\title{
Large cohort observational study integrates current knowledge on changes in outcome for melanoma brain metastases in tyrosine kinase inhibitor (TKI)-immunotherapy era
}

\author{
Stefano Vagge ${ }^{1}$, Andrea Riccardo Filippi ${ }^{2}$ \\ ${ }^{1}$ Radiation Oncology Department, IRCCS San Martino IST National Cancer Research Institute and University, Genova, Italy; ${ }^{2}$ Radiation Oncology \\ Department, Fondazione IRCSS Policlinico S. Matteo, Pavia, Italy \\ Correspondence to: Andrea Riccardo Filippi, MD. Radiation Oncology Department, Fondazione IRCSS Policlinico S. Matteo, Pavia, Italy. \\ Email: a.filippi@smatteo.pv.it. \\ Comment on: Sloot S, Chen YA, Zhao X, et al. Improved survival of patients with melanoma brain metastases in the era of targeted BRAF and immune \\ checkpoint therapies. Cancer 2018;124:297-305.
}

Submitted Jun 14, 2018. Accepted for publication Jul 02, 2018.

doi: $10.21037 /$ tcr.2018.07.02

View this article at: http://dx.doi.org/10.21037/tcr.2018.07.02

There has been a drastic change in the therapeutic strategy for patients with metastatic melanoma over last 7 years. Since 2011, the US Food and Drug Administration (FDA) has approved a number of drugs for advanced disease, including multi-drug combinations. Overall, the 1-year survival rate shifted from $25 \%$ to an impressive $75 \%$ (1). In large randomized phase III trials (RCTs), targeting mutations in BRAF-MEK-ERK pathway or inhibiting the cytotoxic T lymphocyte antigen-4 (CTLA-4) and the programmed cell death protein 1 (PD-1) have been shown to achieve a high rate of durable response, when used as first, second line and over; this lead to 2-year and 3 -year survival rates above $50 \%$ and between $40 \%$ and $50 \%$ respectively $(2,3)$. While waiting for mature overall survival (OS) data of the newest combinations, we have seen a $25 \%$ of patients with stage IV melanoma being cured with immunotherapy, a previously unobserved result; however, still more than half of patients are going to die for progressive disease, and we need to continuously explore new strategies and combination in order to maximize the long-term cure rate.

The brain is a prominent site of treatment failure for metastatic melanoma, and one-third of patients will develop intra-cranial disease; median survival time for these patients was generally poor, ranging from 3 to 5 months (4). As most of the largest RCTs testing BRAF inhibitors and immunotherapy excluded patients with active melanoma brain metastases (MBM), the real impact of immunotherapy and targeted agents on outcomes of MBM patients is still unclear.

At this regard, Sloot and colleagues (5) recently reported the results of an extensive retrospective cohort study including patients who received systemic therapy for advanced melanoma, where incidence and survival of patients with $M B M$ were investigated and compared with a cohort of melanoma patients without MBM, and authors should be congratulated for their effort in exploring this particular setting providing new data.

Notably, the primary endpoint of this study was to investigate for the possible effect of different regimens on de novo MBM development, in patients either treated with chemotherapy, bio-chemotherapy, IL-2, anti-BRAF agents or immune checkpoint inhibitors. The secondary endpoint was to compare OS in patients with and without MBM and confirm and/or identify new prognostic factors. The main value of the study is that it offers a cross-sectional picture of "real-life" clinical advances for patients with MBM trough the last decades and provides interesting new insights into the natural history of the brain metastases when anti-BRAF and immunotherapy are used.

A large retrospective observational cohort of 1,016 patients treated at the Moffit Cancer Centre in Florida between 2000 and 2012 was analyzed. A total of 1,016 patients were initially evaluated for inclusion into this data set; exclusion criteria were: (I) $<2$ months of followup time (245 patients); (II) no digital records available (116 patients); (III) nonmelanoma cancer diagnosis (40 patients); (IV) multiple melanoma primary tumors (four patients); (V) 
missing date of diagnosis (one patient). Finally, 610 patients have been included for analysis. The majority of them, 367 (60.2\%), did not have or develop MBM, while 243 (39.8\%) had MBM: among them, 166 (68.3\%) developed intracranial disease while on treatment, and 77 (31.7\%) at diagnosis. The first brain failure event was most often detected during the first-line therapy (35.4\%).

Patients have been then stratified into three groups, according to the time when targeted therapies were introduced (2000-2008, 2009-2010 and from 2011 onward). Between 2009 and 2010, an increasing number of checkpoint/targeted therapy trials became available and 2011 was the year ipilimumab and vemurafenib were approved.

First, the results confirmed for MBM few known prognostic factors: a limited number of brain metastases $(\mathrm{n}<4)$, the absence of neurological symptoms and a better Karnofsky performance status (>70-90) all resulted to be associated with longer OS. As expected, a global significant different in OS was observed between patients with and without MBM, with median of 25.9 and 35.5 months, respectively $(\mathrm{P}=0.048)$. Among MBM patients, the probability of survival at 1,2 and 3 years was $79.7 \%$ (95\% CI, 76.3-82.7\%), 60.6\% (95\% CI, 56.3-64.6\%) and $45.9 \%$ (95\% CI, 41.4-50.3\%), respectively. To be noted, median OS was 22.7 months in MBM patients diagnosed from 2011, compared with 8.5 and 7.5 months for MBM patients diagnosed between 2009-2010 and 2000-2008 $(\mathrm{P}=0.0002)$, respectively. In line with these findings, the receipt of BRAF-targeted therapy or immune checkpoint inhibitors or the combination of both were all associated with improved OS when tested at multivariate Cox analysis (see supporting information on table 4). A longer OS was also found for patients who were treated locally at the time of MBM detection, highlighting the impact of local therapies even when combined with more effective systemic agents [hazard ratio (HR) 3.5, $\mathrm{P}=0.00038$ ]. The most frequent treatment for $\mathrm{MBM}$ was radiosurgery [stereotactic radiosurgery (SRS)] (48.6\%), followed by whole-brain radiotherapy (WBRT) (15.6\%), neurosurgery $(15.2 \%)$ and starting/continuation of systemic treatments (2.2\%); 5.4\% received no treatment, and the remainder $(13 \%)$ received a combination of radiosurgery and whole brain irradiation. So, most of the patients received a braindirected radiotherapy upfront (77.2\%).

Focusing on the primary endpoint, the overall incidence of de novo MBM among the whole cohort was $40 \%$, comparable to historical datasets (6). Moreover, despite the higher OS observed for MBM patients diagnosed after 2011, the incidence of de novo MBM did not significantly differ between those receiving either BRAF-targeted agents, ipilimumab or anti PD-1/PD-L1 therapies in comparison with traditional chemotherapy. This finding is somehow confounding, being possibly an effect of a selection bias, as probably the patients included in this observational study who were treated with experimental agents (anti-BRAFMEK or immunotherapy) underwent more extensive follow-up brain imaging in comparison to patients receiving chemotherapy, increasing the detection rate of MBM. Once diagnosed, MBM were more efficiently controlled after 2011, and the effect on survival might be composite, being possibly linked to the higher efficacy of new agents, to advances in brain imaging (use of brain MRI) and/ or to a wider use of local therapies such as radiosurgery through time. These are typical confounding factors of a retrospective analysis and cannot be separately evaluated. The higher efficacy in controlling MBM of both anti-BRAF therapies and immunotherapy was independently shown by three prospective trials, which reported the highest intracranial response rate for MBM so far. The COMBIMB trial (7), a multicohort phase II trial including patients with showed in $B R A F^{V 600 E}$-mutated MBM, achieved an overall response rate (ORR) of 58\% (95\% CI, 46-69\%) and the 6-month progression-free survival (PFS) was $44 \%$ (95\% CI, 32-56\%) in asymptomatic patients receiving dabrafenib and trametinib as first therapy for MBM. Long and colleagues (8) then reported the results of a randomized phase II trial designed with the aim of investigating the intracranial response rate in asymptomatic and immunotherapy naïve MBM treated with the combination of Nivolumab and Ipilimumab vs. Nivolumab alone. The trial showed a substantial efficacy of immunotherapy when used upfront, with a $46 \%$ (95\% CI, 29-63\%) intracranial ORR for the combination vs. $20 \%$ (95\% CI, $7-41 \%)$ for the single agent, respectively, with a remarkable 6-month PFS of $53 \%$ (95\% CI, $38-73 \%$ ). The high response duration achieved by Nivolumab combined with Ipilimumab was also disclosed by the single arm phase II Checkmate 204 trial, with preliminary data reporting a 6-month PFS of $67 \%$ (9). Waiting for the survival results, these trials could establish a new benchmark for this population; however, toxicity is an issue, and the integration with local therapies such as radiosurgery is yet to be explored. At this regard, the significative longer OS in patients primarily treated with local therapy after the diagnosis of MBM is one of the most interesting findings in the article by Sloot $e t$ al. A progressive change occurred in the use of RT for MBM, from palliative WBRT to multiple repeated SRS. Detailed data are lacking on this specific point, as tracking brain progression was beyond the scope of the investigation, but we argue that the combination of multiple SRS and single 
agent immunotherapy, given also potential synergistic effects and safety, is worth of investigation, and prospective trials are ongoing (10). In the meantime, given the large amount of retrospective data indicating the safety and efficacy of this approach (for instance SRS followed by antiPD-1), this strategy is common in many centers worldwide. On the other front, the combination with BRAF inhibitors and SRS is to be regarded with caution, given the higher reported rate of unexpected toxic events (10).

In our opinion, the main message from the observational study by Sloot and collaborators is that the change in outcome for MBM patients achieved after the introduction of targeted therapy and immunotherapy appears clearly evident in "real life"; this very positive phenomenon should be taken into account both at diagnosis and progression, omitting WBRT in favor of more conservative approaches, possibly combining in sequence multiple options and keeping in mind the possible long-term survival as well as the potential treatmentrelated morbidity. Information on the best sequence, and the combined use of local therapies and immunotherapy, will hopefully come from ongoing and future trials.

\section{Acknowledgments}

Funding: None.

\section{Footnote}

Provenance and Peer Review: This article was commissioned and reviewed by the Section Editor Dr. Xianxin Qiu [Shanghai Proton and Heavy Ion Center (SPHIC), a.k.a. the Proton and Heavy Ion Center of Fudan University Shanghai Cancer Center (FUSCC), Shanghai, China].

Conflicts of Interest: Dr. AR Filippi: consulting or advisory role: Astra Zeneca; Speakers' Bureau: MSD, Astra Zeneca. $S$ Vagge has no conflicts of interest to declare.

Ethical Statement: The authors are accountable for all aspects of the work in ensuring that questions related to the accuracy or integrity of any part of the work are appropriately investigated and resolved.

Open Access Statement: This is an Open Access article distributed in accordance with the Creative Commons Attribution-NonCommercial-NoDerivs 4.0 International License (CC BY-NC-ND 4.0), which permits the noncommercial replication and distribution of the article with the strict proviso that no changes or edits are made and the original work is properly cited (including links to both the formal publication through the relevant DOI and the license). See: https://creativecommons.org/licenses/by-nc-nd/4.0/.

\section{References}

1. Schachter J, Ribas A, Long GV, et al. Pembrolizumab versus ipilimumab for advanced melanoma: final overall survival results of a multicentre, randomised, open-label phase 3 study (KEYNOTE-006). Lancet 2017;390:1853-62.

2. Wolchok JD, Chiarion-Sileni V, Gonzalez R, et al. Overall Survival with Combined Nivolumab and Ipilimumab in Advanced Melanoma. N Engl J Med 2017;377:1345-56.

3. Dummer R, Ascierto PA, Gogas HJ, et al. Encorafenib plus binimetinib versus vemurafenib or encorafenib in patients with BRAF-mutant melanoma (COLUMBUS): a multicentre, open-label, randomised phase 3 trial. Lancet Oncol 2018;19:603-15.

4. Davies MA, Liu P, McIntyre S, et al. Prognostic factors for survival in melanoma patients with brain metastases. Cancer 2011;117:1687-96.

5. Sloot $\mathrm{S}$, Chen YA, Zhao X, et al. Improved survival of patients with melanoma brain metastases in the era of targeted BRAF and immune checkpoint therapies. Cancer 2018;124:297-305.

6. Sampson JH, Carter JH Jr, Friedman AH, et al. Demographics, prognosis, and therapy in 702 patients with brain metastases from malignant melanoma. J Neurosurg 1998;88:11-20.

7. Davies MA, Saiag P, Robert C, et al. Dabrafenib plus trametinib in patients with BRAFV600-mutant melanoma brain metastases (COMBI-MB): a multicentre, multicohort, open-label, phase 2 trial. Lancet Oncol 2017;18:863-73.

8. Long GV, Atkinson V, Lo S, et al. Combination nivolumab and ipilimumab or nivolumab alone in melanoma brain metastases: a multicenter randomized phase 2 study. Lancet Oncol 2018;19:672-81.

9. Tawbi HAH, Forsyth PA, Algazi AP, et al. Efficacy and safety of nivolumab (NIVO) plus ipilimumab (IPI) in patients with melanoma (MEL) metastatic to the brain: Results of the phase II study CheckMate 204. J Clin Oncol 2017;35:9507.

10. Trino E, Mantovani C, Badellino S, et al. Radiosurgery/ stereotactic radiotherapy in combination with immunotherapy and targeted agents for melanoma brain metastases. Expert Rev Anticancer Ther 2017;17:347-56.

Cite this article as: Vagge S, Filippi AR. Large cohort observational study integrates current knowledge on changes in outcome for melanoma brain metastases in tyrosine kinase inhibitor (TKI)-immunotherapy era. Transl Cancer Res 2018;7(Suppl 7):S735-S737. doi: 10.21037/tcr.2018.07.02 